\title{
Forbidden Reflection Moiré Patterns in Metal-2D Material Interfaces
}

Kate Reidy ${ }^{1}$, Georgios Varnavides ${ }^{1}$, Joachim Dahl Thomsen ${ }^{1}$, Arthur Blackburn ${ }^{2}$, Thang Pham ${ }^{1}$, Abinash Kumar $^{1}$, James LeBeau ${ }^{1}$ and Frances Ross ${ }^{1}$

${ }^{1}$ Massachusetts Institute of Technology, Cambridge, Massachusetts, United States, ${ }^{2}$ University of Victoria, Victoria, British Columbia, Canada

Moiré patterns are a classical interference phenomenon arising from the superposition of two lattices with slightly different periodicities or rotations, and can be observed by local variations in transmitted electron intensity in HR-TEM and STEM. ${ }^{1}$ While the foundations of rotational epitaxy and moiré patterns have been well studied in the fields of thin-film growth and surface science, ${ }^{2}$ there has recently been a resurgence of interest in these topics and their application to two-dimensional (2D) materials. ${ }^{3,4}$ In particular, specific rotations between stacked 2D layers can enhance interlayer coupling, leading to unexpected properties such as Mott insulator and superconducting phases in twisted 2D bilayers. In such 2D/2D systems, the rotation angle can be tuned during fabrication, ${ }^{5}$ resulting in a variety of possible moiré superlattices.

The use of 3D metallic layers on 2D substrates holds equal promise, with evidence that 2D/3D interlayer coupling and charge transfer can strongly influence the contact resistance, photo-response and high frequency performance of 2D/3D heterostructures. ${ }^{6,7}$ Several metals are known to grow epitaxially on 2D materials, creating well-defined rotation angles at the 2D/3D interface. When epitaxial alignment is obtained, studying these 2D/3D moiré patterns with HRTEM presents new opportunities to quantify the crystal rotation and examine defects in the metallic layer and their relationship to structural features on the $2 \mathrm{D}$ substrate.

In this work we explore, theoretically and experimentally, moiré patterns arising from epitaxially aligned \{111\} triangular Au nanoislands on 2D substrates. The Au is deposited in an ultra-high vacuum (UHV) chamber at $\mathrm{T} / \mathrm{T}_{\mathrm{m}} \sim 0.2$, which results in equilibrium shape Au nanoislands that are flat, triangular, and epitaxially aligned with respect to the underlying 2D substrate. An example is shown in Figure 1(a) for a $\mathrm{hBN}$ substrate. We develop a geometric convolution technique to analytically predict the possible moiré periods, which can be mapped to the wave-vector connecting any pair of spots on the selected area diffraction pattern (SAEDP). ${ }^{4}$ It is interesting to note that the $11 \AA$-period moire pattern visible in Figure 1(a) cannot be explained using the zero order Laue zone (ZOLZ) diffraction spots. Instead, note that the FFT of the sample (Fig. 1(a) inset) suggests the presence of strong $1 / 3\{422\}$ reflections. These "forbidden" spots are present in the Au nanoislands in the [111] zone axis, due to both their finite size in the beam direction and the non-integer number of unit cells. The spots can be indexed to Ewald sphere intersections with the first order Laue zone (FOLZ), and their interference with the ZOLZ spots has been shown to result in HRTEM image contrast, akin to the contrast seen at stepped surfaces. ${ }^{9}$ If we include these $1 / 3\{422\}$ spots in the geometric convolution analysis we can explain the observed $11 \AA$ moiré period by a $20^{\circ}$ rotation with respect to substrate. Further, at this rotation we predict another weaker $19 \AA$-period moiré pattern, consistent with the FFT and geometric convolution analysis (Fig. 1(b)). 
A second example, with Au on $\mathrm{MoS}_{2}$, is shown in Figure 2(a). In this case, the most visible moire period is $18 \AA$. Our calculation shows that this arises from interference with the expected ZOLZ $\{220\}$ Au spots and the $\{2110\}$ of $\mathrm{MoS}_{2}$ (Figs. 2(a,b)). However, close examination of the SAEDP (Fig. 2(c)) shows that a second moiré periodicity of $32 \AA$ should be visible, arising from the forbidden reflection and $\{1010\}$ $\mathrm{MoS}_{2}$ spots, and is correctly identified by our geometric convolution technique (Fig. 2(b)). This forbidden reflection moire superlattice is not visible in conventional HR-TEM (Fig. 2(a)) and STEM (Fig. 2(d)) images due to the overlying stronger $18 \AA$ moiré unit cell. However, we can explore it using annular dark field (ADF) 4D STEM datasets (Figures 2(e-f)).

In summary, we find that $\mathrm{Au}$ nanoislands on $2 \mathrm{D}$ materials produce multiple moiré periodicities with differing levels of contrast, and some of these moirés involve the "forbidden" reflection $1 / 3\{422\}$. The simultaneous analysis of several moiré periods enables rotation angles to be determined without ambiguity. For the particular samples analyzed here, the appearance of the moiré patterns is consistent with epitaxial alignment and no distortion or dislocations in the nanoislands. We suggest that the Au nanoislands are loosely bound to the underlying substrate, a conclusion that is corroborated by island motion that is visible during both STEM imaging at room temperature and under heating in UHV in-situ TEM conditions. We suggest that combined use of 4D STEM data and geometric convolution provides a versatile approach to analyze forbidden and bulk reflection moirés, and will help to explore the implications of interference effects on physical properties at the 2D/3D interface, such as enhanced charge transfer and moiré-modulated electrostatic interactions.
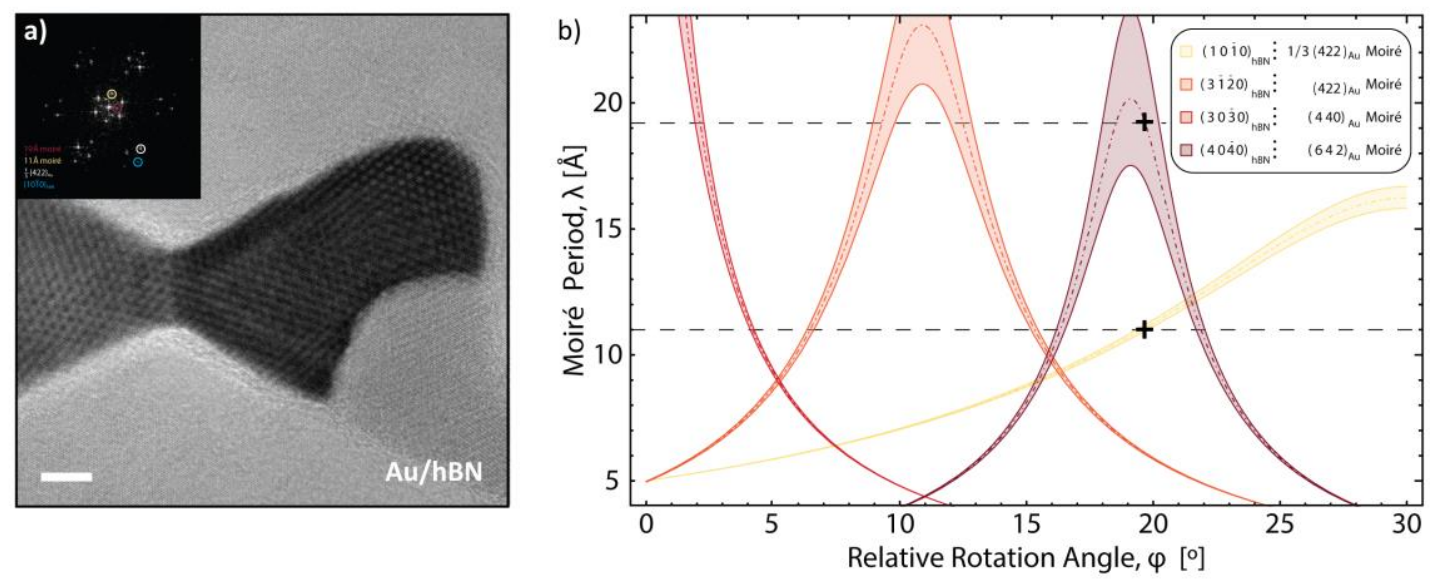

Figure 1. a) HR-TEM image of two $\{111\}$ triangular Au nanoislands on hBN, each showing $11 \AA$-period moiré pattern. Inset shows the FFT of the image, indicating the $1 / 3\{422\}$ forbidden reflection spots, moiré satellite spots, as well as suggesting a larger period moiré. The grey region to the lower right is a nanocrystal of Ge, not analyzed further here. Scale bar $5 \mathrm{~nm}$. b) Subset of possible moiré supercells for $\mathrm{Au}$ on $\mathrm{hBN}$ for every possible rotation angle, determined via the geometric convolution technique. Black dotted lines represent the experimentally observed $(11 \AA)$ and FFT-suggested $(19 \AA)$ moiré periods. Both of these can be explained using a 20 o rotation (markers). 

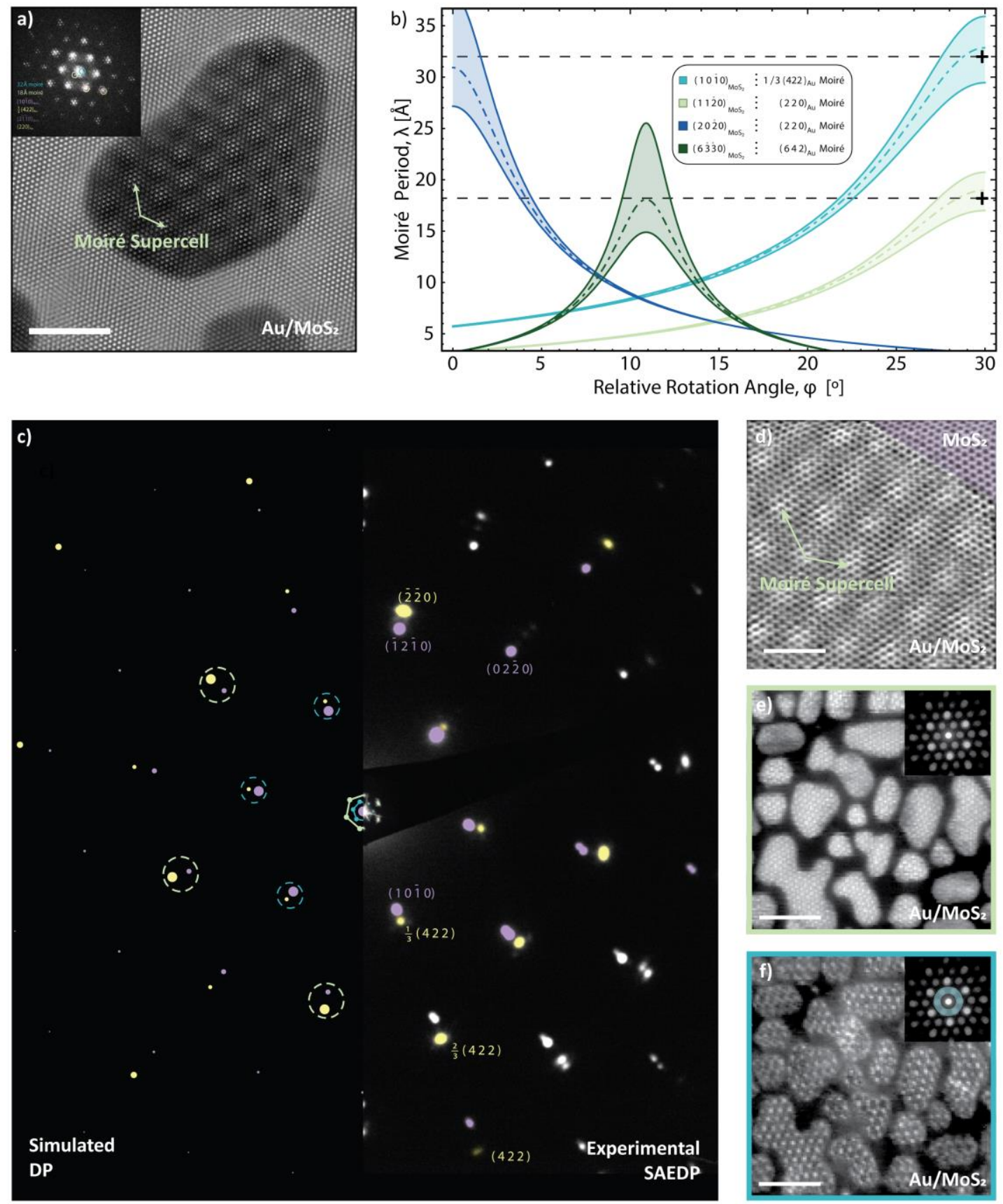

Figure 2. a) HR-TEM image of $\{111\}$ Au nanoislands on MoS2, showing $18 \AA$ A-period moiré pattern. Inset shows the FFT of the sample, highlighting the satellite moiré spots. Scale bar $5 \mathrm{~nm}$. b) Subset of the possible moiré supercells for $\mathrm{Au}$ on $\mathrm{MoS} 2$, determined via the geometric convolution technique. Black dotted lines represent the two experimentally observed moiré periods in the HR-TEM and 4D STEM data. At 30o epitaxial orientation, we predict two moiré periods, at $18 \AA$ and $32 \AA$ (markers). c) Simulated diffraction pattern for $\mathrm{Au}$ and MoS2 (left), compared with the experimentally obtained SAEDP and FFT of the HR-TEM moiré (right). False colour represents: MoS2 (purple), Au (yellow), $18 \AA$ moiré (green) and $32 \AA$ moiré (cyan). Green and cyan circled areas represent spots contributing to $18 \AA$ and $32 \AA$ moirés, respectively. d) Atomic resolution STEM image of Au on MoS2 showing the visible $18 \AA$ moiré supercell. 
Scale bar $2 \mathrm{~nm}$ and uncorrected scan distortion visible near the top of the sample caused by charging. e) 4D STEM image showing the brightest $18 \AA$ A-period moiré, and f) ADF 4D STEM image using the 1/3 $\{422\}$ forbidden reflection spots highlighting weaker $32 \AA$-period moiré. Scale bars $20 \mathrm{~nm}$. Inset shows the 4D STEM DP, alongside the ADF annulus used around $1 / 3\{422\}$ forbidden reflection spots.

\section{References}

1. Oster, G. \& Nishijima, Y. Moiré Patterns. Scientific American 208, 54-63 (1963).

2. Bassett, G. ., Menter, J. . \& Pashley, D. . Moiré patterns on electron micrographs, and their application to the study of dislocations in metals. Proc. R. Soc. London. Ser. A. Math. Phys. Sci. 246, 345-368 (1958). 3. Woods, C. R. et al. Commensurate-incommensurate transition in graphene on hexagonal boron nitride. Nat. Phys. 10, 451-456 (2014).

4. Zeller, P. et al. What are the possible moiré patterns of graphene on hexagonally packed surfaces? Universal solution for hexagonal coincidence lattices, derived by a geometric construction. New J. Phys. 16, (2014).

5. Cao, Y. et al. Unconventional superconductivity in magic-angle graphene superlattices. Nature 556, 43-50 (2018).

6. Sundaram, R. S. et al. The graphene-gold interface and its implications for nanoelectronics. Nano Lett. 11, 3833-3837 (2011).

7. Wang, Y. et al. Van der Waals contacts between three-dimensional metals and two-dimensional semiconductors. Nature 568, 70-74 (2019).

8. Buff, P.-A., Flueli, M., Spycher, R., Stadelmann, P. \& Borelb, J.-P. Crystallographic Structure of Small Gold Particles studied by High-resolution Electron Microscopy. Faraday Discuss 92, (1991).

9. Reyes-Gasga, J., Gómez-Rodríguez, A., Gao, X. \& José-Yacamán, M. On the interpretation of the forbidden spots observed in the electron diffraction patterns of flat $\mathrm{Au}$ triangular nanoparticles. Ultramicroscopy 108, 929-936 (2008). 